\title{
The Kiss-1/Kiss-1R complex as a negative regulator of cell motility and cancer metastasis (Review)
}

\author{
KE JI, LIN YE, MALCOLM D. MASON and WEN G. JIANG \\ Metastasis and Angiogenesis Research Group, Cardiff University School of Medicine, Cardiff, Wales CF14 4XN, UK
}

Received April 20, 2013; Accepted July 19, 2013

DOI: $10.3892 / \mathrm{ijmm} .2013 .1472$

\begin{abstract}
Metastasis is a complex multistep process that involves the impairment of cell-cell adhesion in the neoplastic epithelium, invasion into adjacent tissues and the dissemination of cancer cells through the lymphatic and haematogenous routes. The inhibition of the metastatic process at an early stage has become a hot topic in cancer research. The Kiss-l gene, initially described as a suppressor of metastasis in malignant melanoma, encodes the Kiss-1 protein which can be processed to other peptides, e.g., Kisspeptin-10, Kisspeptin-13, Kisspeptin-14 and Kisspeptin-54. These peptides are endogenous ligands of the Kiss-1 receptor (Kiss-1R), a G protein-coupled receptor (GPR) also known as hOT7T175, AXOR12 or GPR54. The Kiss -1 gene has been suggested as a suppressor of metastasis in a various types of cancer, including gastric cancer, oesophageal carcinoma, pancreatic, ovarian, bladder and prostate cancer, through the regulation of cellular migration and invasion. In the current review, we summarise the current understanding of the role of Kiss-1 and Kiss-1R in cancer and cancer metastasis.
\end{abstract}

\section{Contents}

1. Tumour suppressor genes in cancer

2. Kiss-1

3. Kiss-1 receptor, discovery and structure

4. Kiss-1, Kiss-1 receptor and downstream pathways

5. Kiss-1 and Kiss-1R in cancer

6. Conclusion

\section{Tumour suppressor genes in cancer}

The accumulation of intracellular gene mutation results in neoplastic transformation. The normal or abnormal phenotype

Correspondence to: Professor Wen G. Jiang, Metastasis and Angiogenesis Research Group, Cardiff University School of Medicine, Heath Park, Cardiff, Wales CF14 4XN, UK

E-mail: jiangw@cardiff.ac.uk

Key words: Kiss-1, Kiss-1 receptor, G protein-coupled receptor 54, tumour metastasis suppressor, invasion and migration of a cell is dependent on the cell genotype and intracellular epigenetic alternations. Gene mutations, which include tumour suppressor gene (1), oncogene (e.g., K-ras, $C$-myc and src) and the DNA mismatch repair gene, are the basis of cancerisation (Fig. 1) (2-4).

Tumour suppressor genes maintain cell stability by regulating cell proliferation and differentiation. Therefore, uncontrollable cell proliferation and the formation of tumours may be a result of inactivated tumour suppressor genes caused by somatic mutation or heredity. Adenomatous polyposis coli $(A P C)$ (5) located at chromosome 5q.22.2 and the p53 gene located at chromosome $17 \mathrm{p}$ are possibly the most extensively investigated tumour suppressor genes, both of which are involved in the regulation of the cell cycle, apoptosis, cellular senescence and cellular functions of intestinal cells (e.g., proliferation, differentiation, migration and polarity) (6). Another protein complex that may have tumour and tumour metastasis suppressing functions is Kiss-1 and the Kiss-1 receptor (Kiss-1R).

\section{Kiss-1}

Lee et al (7) introduced the full length of chromosome 6 into the C8161 human metastatic melanoma cell line using a microcell-mediated transferring technique and then the same study group found that the introduction of chromosome 6 suppressed metastasis without affecting tumourigenicity and tumour invasion (8). Two years later, the human Kiss-1 gene was isolated and identified from the melanoma cells by Lee et al (7) and Lee and Welch (9). The premature coding product of the Kiss- 1 gene is a protein with 145 amino acids. The protein is subsequently cleaved into a family of Kisspeptins, including Kisspeptin-10, Kisspeptin-13, Kisspeptin-14 and Kisspeptin-54 $(2,10,11)$. The Kiss-1 gene mapped to chromosome 1q32 was identified as a human melanoma metastasis suppressor gene through the analysis of subtractive hybridisation in highly metastatic cell lines as compared with non-metastatic cell lines (8). These early findings suggested that there was a regulatory gene existing on chromosome 6, which regulates the expression of Kiss-1 gene. Subsequent research revealed that the regulatory region of Kiss-1 was in a locus of a 40-cM region between 6q16.3 and q23 of chromosome 6 (12). A complementary analysis of 51 melanoma patients by Shirasaki et al (13) revealed that the loss of $6 \mathrm{q} 16.3$ - q23 was observed in 14 melanoma patients 


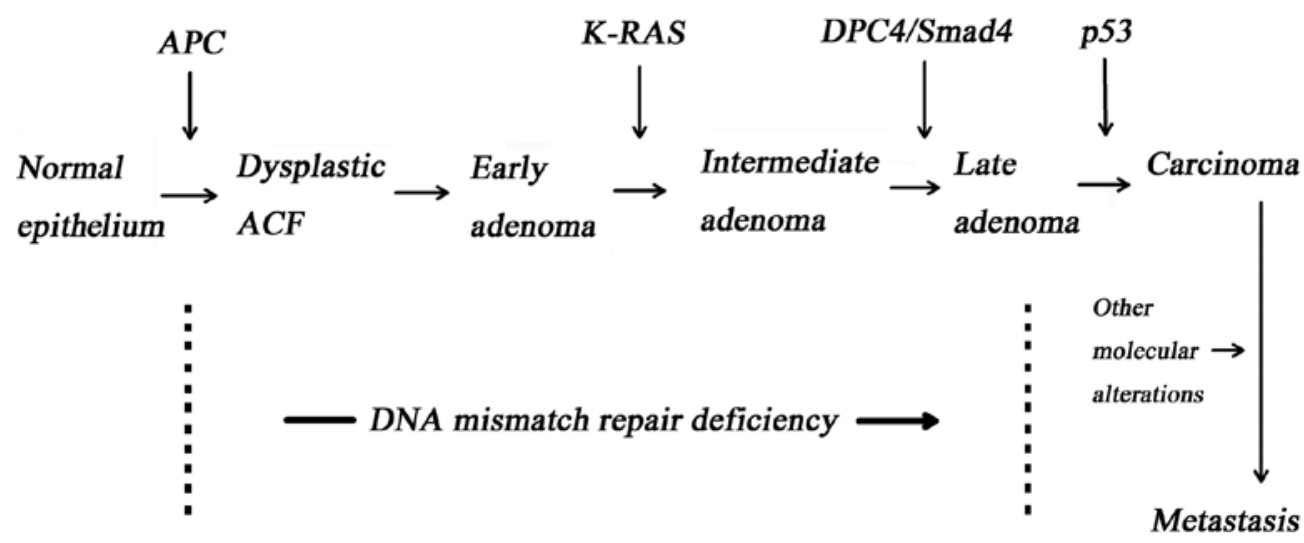

Figure 1. Flow chart demonstrating gene mutations during the process of tumour progression, from adenoma to carcinoma. APC, adenomatous polyposis coli; $\mathrm{ACF}$, aberrant crypt foci; DPC4, deleted in pancreatic cancer locus 4.

(51\%), which had a significant correlation $(\mathrm{P}=0.03)$ with the loss of Kiss-1 expression (44\%). In addition, Goldberg et al (14) found the high expression of thioredoxin interacting protein (TXNIP, also known as vitamin D upregulated protein 1, thioredoxin binding protein 2 or VDUP1) in non-metastatic melanomas, which was mapped to chromosome 1q and also expressed in the neo6/C8161 melanoma cell line. In subsequent studies, the authors reported that increased TXNIP expression inhibited the metastasis of melanoma cells via an upregulation of Kiss-1. Moreover, PCR karyotyping revealed that the expression of Kiss-1 and TXNIP was upregulated in the cells transfected with CRSP3 (vitamin D receptor interacting protein), which was mapped to chromosome 6 and led to a suppression of metastasis (14). The decreased Kiss-1 expression and increased metastasis have been shown to correlate with the loss of CRSP3 (14).

These findings indicate that CRSP3 mapped to chromosome 6 is an upstream regulator of TXNIP, which subsequently regulates the expression of Kiss-1. In other words, a loss of CRSP3 expression, which is caused by the structural abnormality of chromosome 6, impairs the expression of Kiss-1 and TXNIP.

\section{Kiss-1 receptor, discovery and structure}

Kiss-1R, also known as $\mathrm{G}$ protein-coupled receptor 54 (GPR54), hOT7T175, AXOR12 and metastin receptor, was first discovered and cloned from a rat brain in 1999 (15). In humans it was mapped to chromosome 19p13.3 and contains 5 exons and 4 introns and encodes 398 amino acids $(75 \mathrm{kDa})$ and shares $81 \%$ protein homology with the preceding cloned rat orthologue $(11,16)$.

The tissue distribution of Kiss-1 and its receptor are often concordant. For example, Kiss-1 and Kiss-1R are highly expressed in placental tissue $(2,7,16)$. Moreover, Kiss-1 and its receptor are widely distributed throughout the central nervous system (16). High levels of Kiss-1R have been observed in the cerebral vortex, cerebellum, thalamus and pons-medulla (16). By contrast, its cognate ligand precursor, Kiss-1, is highly expressed in the hypothalamus and pituitary gland (11). In addition to placental tissue, the expression of Kiss-1R is also high in the pancreas, whilst it is expressed at low levels in adipose tissue, the lymph nodes, peripheral blood lymphocytes, the pituitary gland and spleen $(2,16)$.

\section{Kiss-1, Kiss-1 receptor and downstream pathways}

The concrete mechanism(s) through which Kiss-1 suppresses tumour metastasis are yet to be established. However, a few signal transduction pathways have been indicated as the key downstream events of the Kiss-1/Kiss-1R complex (Fig. 2).

Calcium mobilisation through $\mathrm{G} q$ activation. Intracellular $\mathrm{Ca}^{2+}$ levels have been shown to significantly increase following transfection with Kiss-1R followed by treatment with Kiss-1 or Kisspeptins in B16-BL6 melanoma (2), CHO-K1 (11) and HEK293 cells (16). Hence, the mobilisation of calcium in cells, when treated with Kiss-1, manifests the important role of Kiss-1R. Kiss-1R is likely coupled to G-proteins of the Gaq/11 subfamily rather than to the Gi or Gs subfamily (16). Through the Gaq/11-mediated signaling pathway, phospholipase $C \beta$ (PLC $\beta$ ) is activated, which consequently generates 2 types of second messengers in cells (inositol triphosphate 3 and diacylglycerol), leading to intracellular $\mathrm{Ca}^{2+}$ release and protein kinase $\mathrm{C}$ activation, respectively (17). Increased intracellular $\mathrm{Ca}^{2+}$ levels markedly suppress tumour metastasis and induce the differentiation and apoptosis of human cancer cells (17). In addition, the capacity of colony formation of breast cancer cells (MDA-MB-435) in hard agar medium has been shown to negatively correlate with the expression of Kiss-1 (18).

Matrix metalloproteinases (MMPs). MMPs can degrade the extracellular matrix and play dominating roles in the process of tumour metastasis. MMP-9, known as the most important protease related with tumour metastasis, is capable of degrading the primary structure of the extracellular matrix and basement membrane (collagen, laminin, fibronectin), and thereby promotes tumour metastasis (19). Yan et al (20) showed that there was a marked reduction in in vitro invasion through Matrigel ${ }^{\circledR}$-coated porous filters by HT-1080 cells which had reduced transcription and activity of MMP-9 following the induced overexpression of Kiss-1. This reduction is credited in part to the diminished $\mathrm{p} 65$ and $\mathrm{p} 50 \mathrm{NF}-\mathrm{\kappa B}$ proteins which interact with the promoter of MMP (17). Consequently, the 


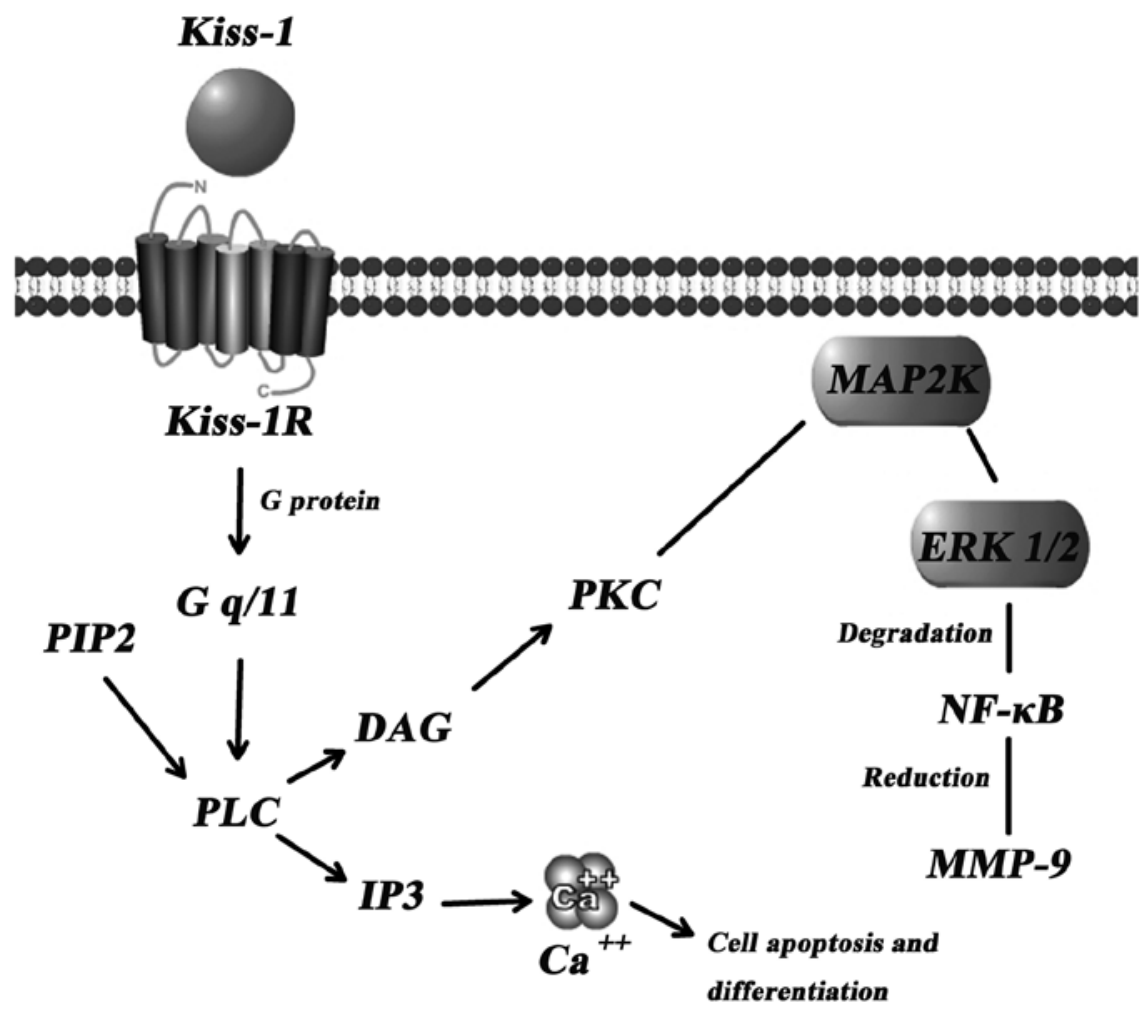

Figure 2. The suggested mechanisms of neuronal depolarisation by Kiss-1 and its receptor (Kiss-1R). Gaq/11 is activated by Kiss-1R and phospholipase C (PLC) to cleave phosphatidylinositol 4,5-bisphosphate (PIP2) into inositol trisphosphate (IP3) and diacylglycerol (DAG). IP3 promotes intracellular calcium ion release inducing cancer cell differentiation and apoptosis, while DAG activates a signaling cascade by activating PKC. The reduction of matrix metalloproteinase (MMP)-9 caused by the degradation of NF- $\mathrm{kB}$ contributes to the suppression of tumour metastasis by activating the MAPK pathway.

reduced synthesis of MMP-9 induces certain inhibitory effects on the mobility and invasion of cancer cells $(2,17)$. In addition, activated focal adhesion kinase (FAK) and paxillin, which play a crucial role in the process of the formation of focal adhesion, exhibit certain links with Kiss-1 and its receptor (21). In ARO thyroid cancer cells transfected with Kiss-1R, Kiss-1 treatment has been shown to elicit a strong and sustained phosphorylation of ERK1/2, and at the same time, a weak phosphorylation of p38 or pAKT (21). A similar concentration-dependent release of arachidonic acid induced by Kisspeptin-10 was observed in CHO-K1/MR cells, in which Kisspeptin-10 markedly inhibited cell proliferation (11). Activated ERK1/2 significantly induces the formation of stress fibres, focal adhesion and activates RHO, via the phosphorylation of FAK and paxillin $(2,21)$.

\section{Kiss-1 and Kiss-1R in cancer}

Breast cancer. Lee and Welch (9) hypothesised that Kiss-1 suppresses metastasis in other tumour types, in addition to melanomas, in which Kiss-1 was discovered. This was on the basis of the map location of the Kiss-1 gene (chromosome 1 bands q32-41), where its 1q alternations are peculiar in a majority of human tumours but not in breast carcinomas. To examine this hypothesis, the authors first transfected full-length Kiss-1 cDNA into a human breast cancer cell line (MDA-MB-435) and then implanted the cells into athymic nude mice with paired control cells. The results demonstrated that the metastatic ability of the transefected cells markedly increased, although tumourigenecity was suppressed (18). To further examine the hypothesis, using RT-PCR, Mitchell et al (22) discovered that the loss of Kiss-1 gene expression was associated directly with the expression levels of activator protein-2a and specificity protein-1, 2 known transcription factors expressed in highly metastatic breast cancer cell lines (22). In addition, Marot et al (23) found low levels of Kiss-1 mRNA in estrogen receptor (ER) $\alpha$-negative MDA-MB-231 cells, whereas ER $\alpha$-positive MCF7 and T47D cells exhibited higher expression levels of Kiss-1 and its receptor. Of note, data from post-menpopausal women with breast cancer showed an opposite trend (23). An analysis of Kiss-1 mRNA in paraffin-embedded stage II or III lymph node-positive breast adenocarcinomas demonstrated that Kiss-1 gene expression was silenced. These observations also support the anti-metastatic potential of Kiss-1 in certain breast cancers (24). Likewise, another study investigated the metastasis-suppressor gene profiles in breast cancer using frozen tissue samples, and analysed Kiss-1 expression at the mRNA and protein levels, using RT-PCR and immunohistochemical staining, respectively (25). The study showed that Kiss-1 mRNA expression was suppressed in samples of brain metastases from breast cancer. In contrast to the studies supporting the anti-metastatic potential of Kiss-1 in breast cancer, Martin et al (26) found that the expression of Kiss-1 was markedly increased in primary tumours compared with normal mammary tissues. The increased expression of Kiss-1 in primary tumours also correlated with metastasis to lymph 
nodes. Likewise, the expression of Kiss-1 increased in relation to tumour grade and increased TMN status. By contrast, its receptor (Kiss-1R) was significantly decreased in patients with poor survival. Moreover, the invasiveness of breast cancer cells was impaired by the knockdown of Kiss-1 (26).

Gastric cancer. Dhar et al (27) found that reduced Kiss-1 expression in gastric cancer correlated with an increased risk of distant metastases and tumour recurrence. The study analysed the expression of Kiss-1 in frozen tissue samples from 40 gastric adenocarcinomas using RNase protection assays, and suggested that Kiss-1 was a possible independent predictor of patient survival compared with the conventional routine prognostic predictors of gastric cancer (27). Likewise, an investigation of 2 metastasis suppressor genes, Kiss-1 and KAI-1 in 49 gastric adenocarcinoma tissues together with 20 pre-cancerous tissues, showed that Kiss-1 mRNA leves were significantly lower in cancer tissues compared with precancerous tissues. This indicates the possible predictive value of Kiss-1 in the prognosis of patients with gastric cancer. Similar results were obtained in the study by Yao et al (28), demonstrating reduced Kiss-1 expression in moderate-to-severe hyperplasia compared with normal-to-moderate hyperplasia, as well as in T3/T4 tumours with lymph node involvement and distant metastases compared with tumours at earlier stages, i.e., T1-2 without any metastasis (28).

Oesophageal carcinoma. Oesophageal squamous cell carcinoma (ESCC) is one of the malignancies for which limited treatment means are available. A number of reasons may contribute to the difficulties in treating this type of tumour, including late diagnosis and advanced stages of the disease at the time of the first visit, commonly ocurring lymph node metastasis in most patients, the anatomical reasons that the organ is closely surrounded by certain key organs and tissues and its rich lymphatic drainage. The latter is of particular interest: even when diagnosed at an early stage, the majority of ESCC patients have already developed lymphatic metastases or lymph node metastasis soon after surgery (29). Therefore, lymph node metastasis is suggested as the most important predictor of prognosis in ESCC. Ikeguchi et al (30) assessed the expression levels of Kiss-1 and Kiss-1R in ESCC tumours and non-cancerous tissues of the oesophagus of 71 patients with ESCC. Using real-time PCR, the authors found that the loss of expression of Kiss-1 and its receptor was detected in $86-100 \%$ of primary tumours with lymph node metastases, suggesting the relevance to lymphatic metastasis and unfavorable prognosis, independent of the depth of tumour invasion (30). Furthermore, the loss of expression of Kiss-1 and its receptor has been observed in primary tumours with invasion to the adventitia compared with the control groups without invasion and has been associated with lymph node metastasis (2). These findings strongly suggest that the loss of expression of one or both genes (Kiss-1 and Kiss-1R) is vital to lymph node metastasis; thus, Kiss-1 and Kiss-1R can be considered as prognostic factors in ESCC (2).

Hepatocellular carcinoma (HCC). A small number of studies on the expression of Kiss-1 are available in the literature regarding tumour thrombus caused by the invasion of HCC to the portal vein as the most vital prognostic factor of orthotopic liver transplantation for HCC (20-70\%) (31). Hou et al (32) reported a correlation between the expression of Kiss-1 and MMP-9 in the formation of portal vein tumour thrombus (PVTT) in HCC by analysing 50 specimens of HCC (31 with PVTT and 19 without PVTT). The expression of Kiss-1 was lower in HCC with PVTT than that in HCC without PVTT. It is noteworthy that a contrasting expression pattern of MMP-9 was observed in HCC with and without PVTT. Hou et al (32) thus suggested that Kiss-1 may possibly prevent PVTT by upregulating MMP-9 expression. Of note, a higher expression of Kiss-1 and Kiss-1R genes was detected in more advanced tumours by Ikeguchi et al (33). This indicates that the expression levels of Kiss-1 and its receptor genes may promote tumour progression rather than suppressing metastasis (33). Similarly, the strong positive immunoreactivity of Kiss-1 and Kiss-1R was also observed in HCC samples which showed higher Kiss-1 expression in HCC cases with poor prognosis (34).

Pancreatic cancer. In pancreatic cancer, the loss of $6 \mathrm{q}, 8 \mathrm{q}$, $9 \mathrm{q}, 17 \mathrm{q}$ and $18 \mathrm{q}$ has been observed and this loss is associated with lymph node and distant metastases, suggesting that there is a vital suppressor gene existing in these regions for pancreatic cancer $(35,36)$. Due to the presence of the Kiss-1 gene in the region, it was suggested that Kiss-1 expression may be downregulated in pancreatic cancer. In a subsequent study employing a number of pancreatic cancer cell lines (AsPC-1, BxPC-3, Capan-2, CFPAC-1, PANC-1 and SUIT-2), Masui et al (37) found that Kiss-1 mRNA levels were reduced in pancreatic cancer, whereas Kiss-1R mRNA expression was higher. In 2007, Liang et al (38) obtained similar results after detecting Kiss-1 expression in pancreatic tumors induced in Sprague-Dawley rats by in situ hybridisation. Lower levels of Kiss-1 mRNA were observed in the pancreatic cancer tissues compared with normal pancreatic tissues, suggesting that Kiss-1 may be an inhibitor of pancreatic cancer metastasis (38).

Thyroid cancer. Distant metastasis is also a crucial factor for the shortened survival of patients suffering from thyroid cancer. Patients who develop distant metastases from thyroid cancer have a 5-year survival rate of approximately 50\% (39). Previous studies have demonstrated the importance of certain receptors involved in the metastatic capacity of thyroid cancer cells. Through the analysis of 36 thyroid tissue samples (13 papillary carcinomas, 10 follicular carcinomas, 2 benign follicular adenomas and 11 normal samples) using real-time PCR, Ringel et al (21) reported that higher levels of expression of Kiss-1 and its receptor were detected in papillary and follicular cancers compared with normal tissue. The increased expression of both gene products was associated with the greater possibility of developing distant metastases (21). Furthermore, the overexpression of Kiss-1R has been shown to result in decreased growth and migration capabilities of thyroid cancer cells following exposure to Kisspeptin-54 (40). The authors found that Kisspeptin-54 increased the protein levels of myocyte-enriched calcineurin interacting protein 1 (MCIP1), which is known as a calcineurin inhibitor. MCIP1 expression has been shown to be increased during the early stages of thyroid tumours and then to be reduced or absent from lymph node metastases (40). 
Ovarian cancer. Ovarian cancer is one of the leading female cancers globally. Data have demonstrated that 24,400 women were newly diagnosed with ovarian cancer and almost $58 \%$ of them died from ovarian cancer in the United States in 2003 (41). Jiang et al (42) measured 7 ovarian cancer cell lines using real-time RT-PCR and reported that the overexpression of Kiss-1 reduced metastatic colony formation by inhibiting cell migration. Similar data were reported by Gao et al (43), who, using immunohistochemical staining, analysed the expression of Kiss-1 protein in 100 primary ovarian epithelial tumours (10 normal tissues, 20 benign adenomas, 20 borderline tumours and 50 malignant tissues). The study demonstrated that there was a significant increase in Kiss-1 expression levels in cancerous tissues compared with benign and normal tissue, wherease increased Kiss- 1 expression in cancer cell lines suppressed the expression of MMP-9 and NF- $\kappa \mathrm{B}$ (43). Furthermore, Hata et al (44) found that the lower expression of Kisspeptin-54 and Kiss-1R correlated with more deteriorative tumours and worse prognosis in 76 epithelial ovarian cancers.

Gestational trophoblastic neoplasia (GTN). All forms of GTN secrete human chorionic gonadotrophin (hCG), which is a helpful marker in the diagnosis, staging and subsequent assessment of the therapeutic response of malignant GTN by monitoring the serum levels of hCG. hCG levels increase and reach a plateau in patients who are developing malignant changes. It is important to detect the serum hCG levels in patients with GTN. However, similar to the problems associated with parallel assays, issues exist regarding false positives caused by heterophile antibodies in commercially available tests (45). The research community remains highly interested, searching for new biomarkers for tumour types. Horikoshi et al (46) showed that there was a significant increase in the concentration of plasma Kiss-1 receptor (Kisspeptin-54) in pregnant females. Dhillo et al (48) assessed the levels of Kisspeptin-54 in GTN by determining plasma Kisspeptin-immunoreactive (Kisspeptin IR) using chromatographic analysis. They found that there was a fluctuation in plasma Kisspeptin-54 levels following chemotherapy. Moreover, they continued to determine the concentration of plasma Kisspeptin IR in female volunteers. Together with hCG, progesterone and estradiol levels were determined in 11 healthy non-pregnant females, 5 healthy females who had previously been pregnant, 13 healthy females in the first trimester of pregnancy, 38 volunteers at 38 weeks of pregnancy and the same females 15 days post-partum and 11 females diagnosed with invasive GTN using radioimmunoassays and chemiluminescent microparticle immunoassays (47). The result revealed that there was a significant positive correlation between plasma Kisspeptin IR and circulating levels of progesterone and oestradiol. This suggests that plasma Kisspeptin levels may be considered as a tumour marker in patients with malignant GTN (48).

Bladder cancer. As the fourth most common malignancy among men, bladder cancer can be classified based on the depth of invasion. Non-muscle-invasion (pTis, pTa and pT1) occupies $75 \%$ of transitional cell carcinomas (TCCs), muscle infiltration (pT2-pT4) occupies 20\% and the remainder TCCs are metastatic at the time of diagnosis (49). The 5-year survival rate is $>90 \%$ for patients presenting with localised TCC, whilst it is only 50 and $10 \%$ for patients diagnosed with regional and distant metastatic disease spread, respectively (49). Similar to the effect of suppressing cancer metastasis by Kiss-1 observed in other solid tumours, the loss of Kiss-1 was observed in advanced bladder tumours (3). Moreover, low levels of Kiss-1 expression are associated with increased histopathological stage, poor tumour cell differentiation and a poor survival rate $(3,50)$.

Prostate cancer. Prostate cancer is the most common malignancy and the second leading cause of cancer-related mortality among men older than 40 years. There are no curative therapies for advanced prostate cancer (PCa) (51). Even if surgical resection is successful, there is a high probability that months or even year later, the majority of patients will develop local recurrence or distant metastases, which are responsible for the majority of PCa-related deaths $(52,53)$. Due to the lack of timeliness of the analysis of the primary tumour size and histology, the information provided by identification and characterisation of molecular signatures is particularly crucial for the diagnosis of PCa (53). The downregulation of Kiss-1 has been shown to correlate with decreased Kiss-1R expression, which is inversely associated with clinical stage and tumour grade. The enforced expression of Kiss-1 has been shown to increase cell sensitisation to anoikis and chemotherapeutic drugs. Kiss-1 suppresses the migration and invasion of prostate cancer cells. Kiss-1 has therefore been suggested as a potential factor for the risk assessment of PCa progression (4).

Endometrial cancer. Uterine corpus cancer is the main cause of malignant gynaecological disease with $>42,000$ cases diagnosed, and is still stably increasing each year in the United States. Cancer cell invasion followed by metastasis profoundly affects patient prognosis and is considered as a vital issue for the improvement of prognosis for females diagnosed with endometrial cancer (54). Kang et al (55) assessed Kiss-1 and its receptor expression in 92 adenocarcinomas of endometrial cancer using IHC staining and real-time PCR. The results revealed that the low expression of Kiss-1 and its receptor was significantly associated with certain well known poor prognostic factors of distant metastasis (invasion into lymphovascular space and deep myometrial invasion) $(54,55)$. Furthermore, based on the study of subcutaneous xenografts established by inoculating Ishikawa cells into female nude mice, they reported that the decreased number of lymph node metastases of Kiss-1R + Ishikawa cells was observed in metastin-10-treated mice, while there was no significant difference in the tumour size between the metastin treated and non-treated groups. This indicates that Kiss- 1 affects the metastatic potential of cancer cells rather than directly inhibiting tumour growth (55).

The expression of Kiss-1 and Kiss-1R manifests relative differences compared with normal tissue in a variety of tumours, as shown by the data in Table I. As these data were collected from different studies, it is difficult to quantitatively analyse differences in the expression of Kiss-1 and its receptor among different types of cancer. However, the differences in expression between Kiss-1 and Kiss-1R in comparison with normal tissues can be studied qualitatively in each group of cancers. The expression of Kiss- 1 and Kiss-1R in these cancers 
Table I. Expression of Kiss-1 and Kiss-1R in cancer tissue compared with corresponding normal or background tissue.

\begin{tabular}{lccc}
\hline Tumour type & Kiss-1 & Kiss-1R & Refs. \\
\hline Breast cancer & $\downarrow / \uparrow$ & $\downarrow / \uparrow$ & $(18,26)$ \\
Gastric cancer & $\downarrow$ & & $(27,28)$ \\
Esophageal carcinoma & $\downarrow$ & $\downarrow$ & $(30)$ \\
Hepatocellular carcinoma & $\uparrow$ & $\uparrow \uparrow$ & $(33)$ \\
Pancreatic cancer & $\downarrow$ & $\uparrow$ & $(37)$ \\
Thyroid cancer & $\uparrow$ & $\uparrow$ & $(21)$ \\
Ovarian cancer & $\downarrow$ & $\uparrow$ & $(2)$ \\
Gestational & & & \\
trophoblastic neoplasia & $\downarrow / \uparrow$ & $\uparrow / \downarrow$ & $(48,60,61)$ \\
Bladder cancer & $\downarrow$ & $\uparrow$ & $(3)$ \\
Prostate cancer & $\downarrow$ & $\downarrow$ & $(4)$ \\
Endometrial cancer & & $\downarrow$ & $(55,62)$ \\
\hline
\end{tabular}

Kiss-1R, Kiss-1 receptor. $\downarrow$, downregulation compared with normal or background tissue; $\uparrow$, upregulation compared with normal or background tissue.

can be generally divided into 2 categories; one expresses Kiss- 1 and Kiss-1R in accord compared with the control group, but not the other. The upregulation and downregulation of Kiss-1 and Kiss-1R expression have been indicated in various malignancies. The reduced expression of Kiss-1 and Kiss-1R has been detected in gastric cancer (without data on Kiss-1R), oesophageal carcinoma, prostate cancer and endometrial cancer (without data on Kiss-1). By contrast, the increased expression of Kiss-1 and its receptor has also been observed in certain solid tumours, including HCC, thyroid cancer and GTN. Of note, several studies have presented evidence of the opposite expression of Kiss-1 and Kiss-1R in various types of cancer (e.g., breast cancer and gestational trophoblastneoplasia). Additionally, the opposite expression of Kiss-1 compared with that of Kiss-1R has been reported in pancreatic, ovarian and bladder cancer. In addition, Table II demonstrates the negative regulatory effects of Kisspeptin-10 on the motility of several cancer cell types compared with control cells (e.g., breast, pancreatic, ovarian, prostate and endometrial cancer) $(2,4,18,37,42,55)$.

\section{Conclusion}

In conclusion, although it remains controversial to some degree, Kiss-1 has been demonstrated as a suppressor of metastasis in the majority of cancers, including gastric cancer, oesophageal carcinoma, pancreatic, ovarian, bladder and prostate cancer. Correlations between the reduced expression of Kiss-1 and poor clinical outcomes have been evident in the majority of malignancies that have been investigated. A possible explanation for the role played by Kiss-1 in cancer biology can be extrapolated from the correlation between Kiss-1 and MMPs, particularly MMP-9 and MMP-2, whose significance in tumour invasion and metastasis formation is
Table II. Negative regulatory effects of Kisspeptin-10 on the motility of several cancer cell types compared with control cells.

Cell/tumour

types Migration Invasion Proliferaiton Refs.

\begin{tabular}{lcccc}
\hline Breast cancer & & $\downarrow$ & None & $(2,18)$ \\
Pancreatic cancer & $\downarrow$ & None & None & $(37)$ \\
Ovarian cancer & $\downarrow$ & & & $(42)$ \\
Prostate cancer & $\downarrow$ & $\downarrow$ & & $(4)$ \\
Endometrial cancer & $\downarrow$ & & None & $(55)$ \\
\hline
\end{tabular}

$\downarrow$, decrease compared with control cells; None, no effect.

well known (56). Kiss-1 has been reported to negatively regulate MMP-9 expression in ovarian tumours (43). Gao et al reported that Kiss-1 protein formed a steady complex with pro-MMP-2 and pro-MMP-9, which possibly had an effect on the proteolytic processing of Kiss-1 rather than the pro-MMP processing (43). However, there are several reports suggesting that Kiss-1 plays contrasting roles in certain types of cancer (e.g., HCC and breast cancer) $(26,33)$. In these cases it is believed that the relative expression of Kiss-1 and its receptor may be the determining factor. The reason for the contrasting effect in the expression of Kiss-1 and its receptor on the progression of HCC and breast cancer in comparison with other malignant tumours remains unknown, whereas the existence of a correlation between Kiss-1 expression and the hormonal environment has been suggested. Gottsch et al (57) reported that the role played by the protein product of Kiss-1 and Kiss-1R was a vital part of the regulation of gonadotropin releasing hormone $(\mathrm{GnRH})$ secretion. Neurons expressing Kiss-1 can regulate the release of GnRH via Kiss-1R in an autocrine or paracrine manner, thus regulating the release of luteinizing hormone ( $\mathrm{LH})$ /follicle-stimulating hormone (FSH) by effecting the pituitary gland; the expression of Kiss-1 increases through the feedback regulation of high estrogen levels (57). Hence, patients with HCC suffering from liver cirrhosis, which leads to a disturbed hormonal balance, generally show high estrogen levels, and the hyperestrogenic state activate $\mathrm{ER} \alpha$, which binds to the Kiss- 1 gene promoter, in turn elevating Kiss-1 expression (58). The possible correlation between Kiss-1 expression and estrogen levels may also be relevant to breast cancer, since blocking the ER pathway has been suggested as one of the most significant systemic therapies in breast cancer (34). A role played by Kiss-1 and Kiss-1R in other types of cancer regulated by hormones has also been suggested. The increasing expression of Kiss-1 has been reported to suppress the metastasis of SKOV3 ovarian cancer cells through the reverse effects caused by protein kinase $\mathrm{C} \alpha(\mathrm{PKC} \alpha)(42)$. Consistently, this effect has also been observed in melanoma cells (59). Taken together, the data presented in this review demonstrate that the Kiss-1/Kiss1R complex is an interesting molecular complex that has significant prognostic and potential therapeutic value in cancer. The Kiss-1/Kiss-1R complex is also an intriguing complex in cells and cell signaling; its precise mechanisms of action warrant further investigation. 


\section{Acknowledgements}

The authors wish to thank the Albert Hung Foundation and Cancer Research Wales for supporting their study. Dr K.J. is a recipient of the China Medical Scholarship of Cardiff University.

\section{References}

1. McKay JA, Williams EA and Mathers JC: Effect of maternal and post-weaning folate supply on gene-specific DNA methylation in the small intestine of weaning and adult apc and wild type mice. Front Genet 2: 23, 2011.

2. Ohtaki T, Shintani Y, Honda S, et al: Metastasis suppressor gene Kiss-1 encodes peptide ligand of a G-protein-coupled receptor. Nature 411: 613-617, 2001.

3. Sanchez-Carbayo M, Schwarz K, Charytonowicz E, Cordon-Cardo C and Mundel P: Tumor suppressor role for myopodin in bladder cancer: loss of nuclear expression of myopodin is cell-cycle dependent and predicts clinical outcome. Oncogene 22: 5298-5305, 2003.

4. Wang H, Jones J, Turner T, et al: Clinical and biological significance of KISS1 expression in prostate cancer. Am J Pathol 180: 1170-1178, 2012.

5. Cutait R, Calache JE, Borges JL, et al: The value of colonoscopy in low digestive hemorrhages of unexplained cause: analysis of 132 patients. Rev Paul Med 96: 66-68, 1980 (In Portuguese).

6. Clevers H: Wnt/beta-catenin signaling in development and disease. Cell 127: 469-480, 2006.

7. Lee JH, Miele ME, Hicks DJ, et al: KiSS-1, a novel human malignant melanoma metastasis-suppressor gene. J Natl Cancer Inst 88: 1731-1737, 1996.

8. Welch DR, Chen P, Miele ME, et al: Microcell-mediated transfer of chromosome 6 into metastatic human C8161 melanoma cells suppresses metastasis but does not inhibit tumorigenicity. Oncogene 9: 255-262, 1994.

9. Lee JH and Welch DR: Identification of highly expressed genes in metastasis-suppressed chromosome 6/human malignant melanoma hybrid cells using subtractive hybridization and differential display. Int J Cancer 71: 1035-1044, 1997.

10. Bilban M, Ghaffari-Tabrizi N,Hintermann E, et al: Kisspeptin-10, a $\mathrm{KiSS}-1 /$ metastin-derived decapeptide, is a physiological invasion inhibitor of primary human trophoblasts. J Cell Sci 117: 1319-1328, 2004.

11. Kotani M, Detheux M, Vandenbogaerde A, et al: The metastasis suppressor gene KiSS-1 encodes kisspeptins, the natural ligands of the orphan $\mathrm{G}$ protein-coupled receptor GPR54. J Biol Chem 276: 34631-34636, 2001.

12. Miele ME, Jewett MD, Goldberg SF, et al: A human melanoma metastasis-suppressor locus maps to 6q16.3-q23. Int J Cancer 86 $524-528,2000$

13. Shirasaki F, Takata M, Hatta N and Takehara K: Loss of expression of the metastasis suppressor gene KiSS1 during melanoma progression and its association with $\mathrm{LOH}$ of chromosome 6q16.3-q23. Cancer Res 61: 7422-7425, 2001.

14. Goldberg SF, Miele ME, Hatta N, et al: Melanoma metastasis suppression by chromosome 6: evidence for a pathway regulated by CRSP3 and TXNIP. Cancer Res 63: 432-440, 2003.

15. Lee DK, Nguyen T, O'Neill GP, et al: Discovery of a receptor related to the galanin receptors. FEBS Lett 446: 103-107, 1999.

16. Muir AI, Chamberlain L, Elshourbagy NA, et al: AXOR12, a novel human $\mathrm{G}$ protein-coupled receptor, activated by the peptide KiSS-1. J Biol Chem 276: 28969-28975, 2001.

17. Hori A, Honda S, Asada M, et al: Metastin suppresses the motility and growth of $\mathrm{CHO}$ cells transfected with its receptor. Biochem Biophys Res Commun 286: 958-963, 2001.

18. Lee JH and Welch DR: Suppression of metastasis in human breast carcinoma MDA-MB-435 cells after transfection with the metastasis suppressor gene, KiSS-1. Cancer Res 57: 2384-2387, 1997.

19. Lee KH and Kim JR: Kiss-1 suppresses MMP-9 expression by activating p38 MAP kinase in human stomach cancer. Oncol Res 18: 107-116, 2009.

20. Yan C, Wang H and Boyd DD: KiSS-1 represses 92-kDa type IV collagenase expression by down-regulating NF-kappa B binding to the promoter as a consequence of Ikappa Balpha-induced block of p65/p50 nuclear translocation. J Biol Chem 276: 1164-1172, 2001.
21. Ringel MD, Hardy E, Bernet VJ, et al: Metastin receptor is overexpressed in papillary thyroid cancer and activates MAP kinase in thyroid cancer cells. J Clin Endocrinol Metab 87: 2399,2002

22. Mitchell DC, Stafford LJ, Li D, Bar-Eli M and Liu M: Transcriptional regulation of KiSS-1 gene expression in metastatic melanoma by specificity protein-1 and its coactivator DRIP-130. Oncogene 26: 1739-1747, 2007.

23. Marot D, Bieche I, Aumas C, et al: High tumoral levels of Kiss1 and G-protein-coupled receptor 54 expression are correlated with poor prognosis of estrogen receptor-positive breast tumors. Endocr Relat Cancer 14: 691-702, 2007.

24. Kostadima L, Pentheroudakis $\mathrm{G}$ and Pavlidis N: The missing kiss of life: transcriptional activity of the metastasis suppressor gene KiSS1 in early breast cancer. Anticancer Res 27: 2499-2504, 2007.

25. Stark AM, Tongers K, Maass N, Mehdorn HM and Held-Feindt J: Reduced metastasis-suppressor gene mRNA-expression in breast cancer brain metastases. J Cancer Res Clin Oncol 131: 191-198, 2005.

26. Martin TA, Watkins G and Jiang WG: KiSS-1 expression in human breast cancer. Clin Exp Metastasis 22: 503-511, 2005.

27. Dhar DK, Naora H, Kubota H, et al: Downregulation of KiSS-1 expression is responsible for tumor invasion and worse prognosis in gastric carcinoma. Int J Cancer 111: 868-872, 2004.

28. Yao HL, Yang ZL, Li YG and Liu GW: In situ hybridization study on the expression of Kiss-1 and KAI-1 metastasis suppressor genes in gastric cancer. Zhonghua Wei Chang Wai Ke Za Zhi 10: 274-277, 2007 (In Chinese).

29. Tachibana M, Kinugasa S, Dhar DK, et al: Prognostic factors in $\mathrm{T} 1$ and T2 squamous cell carcinoma of the thoracic esophagus. Arch Surg 134: 50-54, 1999.

30. Ikeguchi M, Yamaguchi K and Kaibara N: Clinical significance of the loss of KiSS-1 and orphan G-protein-coupled receptor (hOT7T175) gene expression in esophageal squamous cell carcinoma. Clin Cancer Res 10: 1379-1383, 2004.

31. Cedrone A, Rapaccini GL, Pompili M, et al: Portal vein thrombosis complicating hepatocellular carcinoma. Value of ultrasound-guided fine-needle biopsy of the thrombus in the therapeutic management. Liver 16: 94-98, 1996.

32. Hou YK, Wang Y, Cong WM and Wu MC: Expression of tumor metastasis-suppressor gene KiSS-1 and matrix metalloproteinase-9 in portal vein tumor thrombus of hepatocellular carcinoma. Ai Zheng 26: 591-595, 2007 (In Chinese).

33. Ikeguchi M, Hirooka Y and Kaibara N: Quantitative reverse transcriptase polymerase chain reaction analysis for KiSS-1 and orphan G-protein-coupled receptor (hOT7T175) gene expression in hepatocellular carcinoma. J Cancer Res Clin Oncol 129: 531-535, 2003.

34. Schmid K, Wang X, Haitel A, et al: KiSS-1 overexpression as an independent prognostic marker in hepatocellular carcinoma: an immunohistochemical study. Virchows Arch 450: 143-149, 2007.

35. Rigaud G, Moore PS, Zamboni G, et al: Allelotype of pancreatic acinar cell carcinoma. Int J Cancer 88: 772-777, 2000.

36. Yatsuoka T, Sunamura M, Furukawa T, et al: Association of poor prognosis with loss of $12 \mathrm{q}, 17 \mathrm{p}$, and $18 \mathrm{q}$, and concordant loss of $6 \mathrm{q} / 17 \mathrm{p}$ and $12 \mathrm{q} / 18 \mathrm{q}$ in human pancreatic ductal adenocarcinoma. Am J Gastroenterol 95: 2080-2085, 2000.

37. Masui T, Doi R, Mori T, et al: Metastin and its variant forms suppress migration of pancreatic cancer cells. Biochem Biophys Res Commun 315: 85-92, 2004.

38. Liang S and Yang ZL: Expression of KiSS-1mRNA in pancreatic ductal adenocarcinoma and non-cancerous pancreatic tissues in SD rats. Zhong Nan Da Xue Xue Bao Yi Xue Ban 32: 109-113, 2007 (In Chinese).

39. Singer PA, Cooper DS, Daniels GH, et al: Treatment guidelines for patients with thyroid nodules and well-differentiated thyroid cancer. American Thyroid Association. Arch Intern Med 156: 2165-2172, 1996.

40. Stathatos N, Bourdeau I, Espinosa AV, et al: KiSS-1/G protein-coupled receptor 54 metastasis suppressor pathway increases myocyte-enriched calcineurin interacting protein 1 expression and chronically inhibits calcineurin activity. J Clin Endocrinol Metab 90: 5432-5440, 2005.

41. Jemal A, Murray T, Samuels A, Ghafoor A, Ward E and Thun MJ: Cancer statistics, 2003. CA Cancer J Clin 53: 5-26, 2003.

42. Jiang Y, Berk M, Singh LS, et al: KiSS1 suppresses metastasis in human ovarian cancer via inhibition of protein kinase $\mathrm{C}$ alpha. Clin Exp Metastasis 22: 369-376, 2005. 
43. Gao GL, Liu LD, Zou XS and Chen WX: Expression of KiSS-1, matrix metalloproteinase-9, nuclear factor-kappaBp65 in ovarian tumour. Zhonghua Fu Chan Ke Za Zhi 42: 34-38, 2007 (In Chinese).

44. Hata K, Dhar DK, Watanabe Y, Nakai H and Hoshiai H: Expression of metastin and a G-protein-coupled receptor (AXOR12) in epithelial ovarian cancer. Eur J Cancer 43: 1452-1459, 2007

45. Cole LA: Phantom hCG and phantom choriocarcinoma. Gynecol Oncol 71: 325-329, 1998.

46. Horikoshi Y, Matsumoto H, Takatsu Y, et al: Dramatic elevation of plasma metastin concentrations in human pregnancy: metastin as a novel placenta-derived hormone in humans. J Clin Endocrinol Metab 88: 914-919, 2003.

47. Cole LA and Sutton JM: Selecting an appropriate hCG test for managing gestational trophoblastic disease and cancer. J Reprod Med 49: 545-553, 2004.

48. Dhillo WS, Savage P, Murphy KG, et al: Plasma kisspeptin is raised in patients with gestational trophoblastic neoplasia and falls during treatment. Am J Physiol Endocrinol Metab 291: E878-E884, 2006.

49. Jemal A, Siegel R, Ward E, et al: Cancer statistics, 2008. CA Cancer J Clin 58: 71-96, 2008.

50. Nicolle G, Comperat E, Nicolaïew N, Cancel-Tassin G and Cussenot O: Metastin (KISS-1) and metastin-coupled receptor (GPR54) expression in transitional cell carcinoma of the bladder. Ann Oncol 18: 605-607, 2007.

51. Buchan NC and Goldenberg SL: Intermittent androgen suppression for prostate cancer. Nat Rev Urol 7: 552-560, 2010.

52. Ramiah V, George DJ and Armstrong AJ: Clinical endpoints for drug development in prostate cancer. Curr Opin Urol 18: 303-308, 2008.

53. Plantade A, Massard C, de Crevoisier R and Fizazi K: Locally advanced prostate cancer: definition, prognosis and treatment. Bull Cancer 94: F50-F61, 2007 (In French).
54. Creasman WT, Odicino F, Maisonneuve P, et al: Carcinoma of the corpus uteri. FIGO 26th Annual Report on the Results of Treatment in Gynecological Cancer. Int J Gynaecol Obstet 95 (Suppl 1): S105-S143, 2006

55. Kang HS, Baba T, Mandai M, et al: GPR54 is a target for suppression of metastasis in endometrial cancer. Mol Cancer Ther 10: 580-590, 2011.

56. Nomura H, Sato H, Seiki M, Mai M and Okada Y: Expression of membrane-type matrix metalloproteinase in human gastric carcinomas. Cancer Res 55: 3263-3266, 1995.

57. Gottsch ML, Clifton DK and Steiner RA: Kisspepeptin-GPR54 signaling in the neuroendocrine reproductive axis. Mol Cell Endocrinol 254-255: 91-96, 2006.

58. Li D, Mitchell D, Luo J, et al: Estrogen regulates KiSS1 gene expression through estrogen receptor alpha and SP protein complexes. Endocrinology 148: 4821-4828, 2007.

59. Dissanayake SK, Wade M, Johnson CE, et al: The Wnt5A/protein kinase $\mathrm{C}$ pathway mediates motility in melanoma cells via the inhibition of metastasis suppressors and initiation of an epithelial to mesenchymal transition. J Biol Chem 282: 17259-17271, 2007.

60. Ohta S, Lai EW, Pang AL, et al: Downregulation of metastasis suppressor genes in malignant pheochromocytoma. Int J Cancer 114: 139-143, 2005.

61. Janneau JL, Maldonado-Estrada J, Tachdjian G, et al: Transcriptional expression of genes involved in cell invasion and migration by normal and tumoral trophoblast cells. J Clin Endocrinol Metab 87: 5336-5339, 2002.

62. Jiang T, Zhang SL, Lin B, Meng LR and Gao H: Expression and clinical significance of KISS-1 and GPR54 mRNA in endometrial carcinoma. Zhonghua Zhong Liu Za Zhi 27: 229-231, 2005 (In Chinese). 